\title{
PROPOSIÇÃO DO MODELO DTC PARA GESTÂO DO CONHECIMENTO EM EMPRESAS DE CONSULTORIA EM TECNOLOGIA DE INFORMAÇÃO
}

\author{
PROPOSITION OF DTC MODEL FOR KNOWLEDGE \\ MANAGEMENT IN INFORMATION TECHNOLOGY \\ CONSULTING ENTERPRISES
}

\author{
Data de submissão: 23-09-2013 \\ Aceite: 19-05-2014 \\ Paulo Sergio Gonçalves de Oliveira ${ }^{1}$ \\ Meire dos Santos Lopes ${ }^{2}$ \\ Luciano Ferreira da Silva ${ }^{3}$ \\ Dirceu da Silva ${ }^{4}$
}

\section{RESUMO}

Este artigo teve como objetivo propor, dentro do modelo de gestão do conhecimento, as principais práticas que promovem a aprendizagem dos profissionais que atuam na área de Tecnologia da Informação (TI). A base teórica para este estudo consistiu na validação de um modelo proposto pelos autores, que se baseou no modelo de aprendizagem cunhado por Peter Senge, em seu livro "A Quinta Disciplina", cuja abordagem destaca que, para a aprendizagem organizacional, cinco disciplinas são importantes: pensamento sistêmico, domínio pessoal, modelos mentais, construção de uma visão compartilhada e aprendizagem em equipe. Além do modelo proposto por Senge (2001), este estudo destaca a importância das comunidades de práticas na transferência do conhecimento como uma maneira de promover a aprendizagem individual e organizacional. O modelo estatístico destacou o domínio pessoal, a transferência do conhecimento e as comunidades de prática como os principais fatores para a aprendizagem desses profissionais, fato que motivou os pesquisadores a chamarem o modelo de DTC. Portanto, este estudo se constitui em uma ferramenta para empresas ou pesquisadores que desejam melhorar as práticas adotadas para a aprendizagem organizacional.

Palavras-chaves: Gestão do Conhecimento. Comunidades de Prática. Tecnologia da Informação. PLS-PM. Aprendizagem Organizacional.

\footnotetext{
${ }^{1}$ Possui graduação em Administração pela Faculdade de Informática e Administração Paulista, FIAP, mestrado em Administração pela Universidade Municipal de São Caetano do Sul, USCS e doutorado em Engenharia de Produção pela Universidade Metodista de Piracicaba, UNIMEP. Atualmente é consultor na empresa Indra Consulting, atuando no cliente Mapfre como Administrador de dados e gestão de qualidade. Também é professor de graduação e Pós-graduação (Mestrado em Administração na Universidade Ibirapuera). São Paulo. São Paulo. Brasil. E-mail: psgoliveira@hotmail.com

2 Possui graduação em Administração pela Faculdade de Informática e Administração Paulista e mestrado em Administração pela Universidade Municipal de São Caetano do Sul, USCS. São Caetano do Sul. São Paulo. Brasil. E-mail: meiresl@uninove.br

3 Possui graduação em Administração de Empresas pela Faculdade Brasileira de Recursos Humanos, FBRH, mestrado em Educação, Administração e Comunicação pela Universidade São Marcos, UNIMARCO e doutorado em andamento em Administração pela Pontifícia Universidade Católica de São Paulo, PUC/SP. São Paulo. São Paul. Brasil. E-mail: If_ferreirabr@yahoo.com.br

${ }^{4}$ Possui graduação em Física pela Universidade de São Paulo, USP, mestrado em Física pela Universidade de São Paulo, USP e doutorado em Educação pela Universidade de São Paulo, USP. Atualmente é professor do Programa de Pós-Graduação em Administração da Universidade Nove de Julho e da Universidade Estadual de Campinas. São Paulo. São Paulo. Brasil. E-mail. dirceuds@gmail.com
} 


\begin{abstract}
This paper aims to purpose inside knowledge management framework the main practices to promote information technology professionals learning. The theoretical basis for this study was Organizational Learning models by Peter Senge (2001). This model correlates communities of practice and knowledge transfer models to promote individual and organizational learning these aspects motivates researchers to namely the model as DTC. The objective was achieved using a survey developed with 164 TI Professionals which works in consulting firms in Sao Paulo Regions using PLS-PM statistical tool which determinates the variables are import for professionals learning. The statistical model highlighted Personal Mastery, Knowledge Transfer and Communities of Practices as main factors for this TI professionals learning and knowledge exchange, these aspects motivates the researchers to put the name DTC in model. By the way this study is an important tool for enterprises or researchers which wants to import organizational learning practices using knowledge management framework.

Keywords: Knowledge Management. Communities of Practice. Information Technology. PLSPM. Organizational Learning.
\end{abstract}

\title{
1 INTRODUÇÃO
}

A gestão do conhecimento destaca-se na atualidade como um modelo de gestão que permite às empresas contemporâneas se tornarem mais competitivas neste ambiente de mudanças constantes e geralmente imprevisíveis. As empresas de Tecnologia da Informação (TI) buscam, neste modelo de gestão, as práticas necessárias para sua sobrevivência, razão pela qual o objetivo deste estudo foi desenvolver um modelo de gestão do conhecimento cujas práticas indiquem ações que as empresas de TI possam adotar para promover a aprendizagem e, assim, continuarem competitivas no mercado.

A gestão do conhecimento é um modelo que tem como foco gerenciar o conhecimento individual e organizacional de forma a transformar esses conhecimentos em produtos e serviços que possam agregar valor para a organização. Trata-se de uma abordagem complexa, que aponta várias práticas para a construção, aquisição, disseminação e retenção de conhecimento.

Portanto, tendo em vista a complexidade e variedade de práticas adotadas neste modelo de gestão, este estudo se concentra nas práticas organizacionais voltadas para a aprendizagem nas empresas de Tl, destacando a aprendizagem organizacional, a transferência do conhecimento e as comunidades de prática como principais condutas da gestão do conhecimento a serem adotadas pelas empresas que têm como objetivo promover a aprendizagem, gerando novos conhecimentos para agregar valor à organização e à sociedade, garantindo, assim, sua sobrevivência e competitividade.

\section{REVISÃO BIBLIOGRÁFICA: GESTÃO DO CONHECIMENTO}

Devido à gestão do conhecimento ser uma abordagem composta de várias práticas organizacionais, a revisão bibliográfica está dividida em três subcapítulos, expostos a seguir.

\subsection{Aprendizagem organizacional}

As organizações na atualidade enfrentam um ambiente competitivo, em que é preciso investir na aprendizagem contínua. Para isso, faz-se necessário investir no aprendizado individual e coletivo, melhorando, assim, a memória organizacional (ASLAM et al., 2011). 
A aprendizagem coletiva é atualmente conhecida como aprendizagem organizacional. As ideias a respeito das organizações que aprendem estão presentes na literatura há décadas. No entanto, tais conceitos se tornaram amplamente reconhecidos na década de 1990, mais especificamente com o lançamento do livro "A Quinta Disciplina", de Peter Senge, no qual o autor aponta cinco disciplinas que ajudam as organizações a inovarem (MOHANTY; KAR, 2012). Segundo Peter Senge (2001), as cinco disciplinas que promovem a aprendizagem são:

1. pensamento sistêmico - como exemplo de pensamento sistêmico pode-se citar a tempestade, que fornece sinais tais como o céu escurecer e as folhas girarem no chão. Porém, sabemos que, depois da tempestade, a água da chuva alimentará os lençóis d'água, a quilômetros de distância, e o céu estará claro outra vez. Estes eventos estão todos distantes no tempo e no espaço, mas estão conectados a um padrão. As empresas e os outros seres humanos também são sistemas, ou seja, estão conectados por fios invisíveis de ações inter-relacionadas, que muitas vezes levam anos para manifestar seus efeitos uns sobre os outros;

2. domínio pessoal - a palavra domínio pode sugerir controle sobre as pessoas ou sobre as coisas, mas pode significar um nível especial de proficiência. As pessoas com um alto nível de domínio pessoal conseguem realizar mais rapidamente aquilo que é mais importante para elas. Fazem isso se comprometendo com seu próprio aprendizado ao longo da vida. Pode-se concluir que a capacidade e o comprometimento de uma organização em aprender não devem ser maiores que a de seus integrantes. Porém, surpreendentemente poucas organizações estimulam o crescimento de seus funcionários, o que representa um desperdício de recursos;

3. modelos mentais - são pressupostos profundamente arraigados, generalizações ou mesmo imagens que influenciam nossa forma de ver o mundo e de agir. Na maioria das vezes, não estamos conscientes de nossos modelos mentais ou de seus efeitos sobre o nosso comportamento. Eles incluem também as conversas ricas em aprendizado que equilibrem indagação e argumentação, em que as pessoas exponham de forma eficaz seus próprios pensamentos e estejam abertas à influência dos outros;

4. construção de uma visão compartilhada - é difícil pensar em uma organização que tenha mantido uma posição de grandeza na ausência de metas, valores e missões profundamente compartilhadas na organização. As grandes organizações, apesar de trabalhar com diferentes produtos ou mercados, conseguem reunir as pessoas em torno de uma identidade e um senso de destino comum. Quando existe uma visão verdadeiramente compartilhada, as pessoas dão tudo de si para aprender e melhorar não porque são obrigadas, mas porque querem. Porém, muitos líderes têm visões pessoais que nunca se traduzem nas visões compartilhadas que impulsionam uma organização. Muitas vezes, a visão compartilhada de uma empresa gira em torno do carisma de um líder ou de uma crise que estimula temporariamente todos. A prática da visão compartilhada envolve habilidades de descobrir imagens do futuro que estimulem o compromisso genuíno e o envolvimento, em vez da mera aceitação;

5. aprendizagem em equipe - esta disciplina começa pelo diálogo, sendo necessário o integrante deixar de lado as ideias preconcebidas e participar de um verdadeiro fluxo de pensar em conjunto. A aprendizagem em equipe é vital, pois as equipes, e não os indivíduos, são a unidade de aprendizagem fundamental nas organizações modernas. 
A aprendizagem organizacional é importante porque possibilita a inovação, a adaptação e a melhora da eficiência e da produtividade e porque seus efeitos podem ser medidos por indicadores financeiros e não financeiros. Nesse sentido, alguns estudos correlacionam os efeitos da aprendizagem organizacional com a capacidade de inovação, como é o caso de uma pesquisa desenvolvida por Ussahawanitchakit (2011) na Tailândia com 121 organizações, a qual demonstrou que a mente aberta e a aprendizagem organizacional têm efeito positivo na geração de inovações.

Outros estudos, tais como o de O'learly e Mortensensen (2011), exploram as complexidades existentes nos relacionamentos entre a aprendizagem organizacional e o desempenho dos colaboradores, partindo do princípio de que o uso de aprendizagem organizacional melhora esse desempenho, mas alertam que tal prática apresenta diversos desafios para os gestores e pesquisadores. Um desses desafios são os riscos interpessoais e culturais do comportamento na aprendizagem, sendo a experimentação e o estudo exploratório fatores muito críticos para o processo. Outro aspecto a ser considerado é o apoio dos lideres de forma a suportar a aprendizagem organizacional (TAHIR et al., 2011).

Na busca pela aprendizagem organizacional, as empresas utilizam múltiplas associações entre os indivíduos para melhorar a produtividade individual e o desempenho em equipe, mas essa estrutura pode criar pressões competitivas entre os membros, o que dificulta a aprendizagem e a produtividade. Para evitar esse problema, deve-se balancear a variedade e o número de membros nas equipes (O'LEARY; MORTENSEN, 2011).

Nesse sentido, as empresas precisam trabalhar seus aspectos culturais, já que, em uma organização que aprende, a cultura influencia a criatividade de grupo e também a liderança transformacional (PHIPPS; PRIETO; VERMA, 2012; LEITE; ALBUQUERQUE, 2013). Esse aspecto é bastante importante, pois competências proativas, futurísticas e estratégicas, essenciais a qualquer organização, são capazes de melhorar a sua flexibilidade e adaptabilidade por meio da meIhora da criação de novos conhecimentos. $O$ conceito de organizações que aprendem possui uma grande aceitação e diferentes abordagens (AKHTAR; KHAN, 2011).

Apesar de existirem diferentes conceitos a respeito da aprendizagem organizacional, alguns fatores contextuais facilitam ou dificultam a aprendizagem nas organizações. Segundo Akhtar e Khan (2011), estes fatores são:

a. segurança psicológica, o que significa que os empregados devem se sentir confortáveis para confrontar as ideias dos gestores e de seus pares, de modo a criar um ambiente que aprecie as diferenças e esteja aberto a novas ideias;

b. geração, coleção, interpretação e disseminação da informação e experimentação de novas ideias, elementos que são essenciais a um processo concreto de aprendizagem;

c. comportamento dos líderes, pois as pessoas se sentem encorajadas a aprender quanto os líderes fornecem oportunidades de falar e facilitar o debate;

As organizações convencionais estão adotando algumas ações no sentido de se tornarem empresas que aprendem, o que se deve ao fato de que, na era da informação, o conhecimento é um importante recurso para obter sustentabilidade e competência em um ambiente de negócios mutável. Os termos aprendizagem organizacional e organizações que aprendem são utilizados de forma intercambiável, mas não são sinônimos. As organizações que aprendem são uma forma de organização, enquanto que a aprendizagem organizacional consiste no processo e nas atividades envolvidas na aprendizagem (MOHANTY; KAR, 2012). 


\subsection{Transferência do conhecimento}

As pessoas obtêm conhecimento por meio de uma variedade de caminhos, o que faz com que a gestão do conhecimento venha ganhando importância para as organizações no que se refere a gerenciar as informações e os conhecimentos necessários, assim como a transferência do conhecimento. A transferência do conhecimento é um aspecto derivado da gestão do conhecimento que amplia as suas ações, pois possibilita que o conhecimento seja distribuído para outras empresas, atingindo áreas geográficas maiores (TSENG et al., 2012).

Para exemplificar tal aspecto, Stacke, Hoffman e Costa (2012) desenvolveram um estudo que analisa a transferência de conhecimento que existe entre as empresas pertencentes a um cluster, o que pode ocorrer de diversas maneiras, embora o modo mais recorrente seja por meio de reuniões esporádicas. O conceito de transferência do conhecimento é derivado dos estudos de Nonaka e Takeuchi (1997) e parte do princípio de que o conhecimento é criado em duas dimensões: a epistemológica e a ontológica. A dimensão epistemológica destaca os conhecimentos tácito e explícito em suas bases conceituais, já a dimensão ontológica aborda a construção do conhecimento a partir do indivíduo até a construção do conhecimento organizacional.

No entanto, as empresas enfrentam dificuldades no que se refere à transferência do conhecimento aos parceiros, valendo-se de estruturas formais e informais para melhorar essa transferência (LICHTENTHALER, 2010; RODRIGUES; PEDRON; OLIVEIRA, 2013). Nesse sentido, o conhecimento tácito é mobilizado e ampliado organizacionalmente por meio de quatro modos de conversão do conhecimento - internalização, externalização, socialização e combinação - e cristalizado em níveis ontológicos superiores, ou seja, no nível corporativo. Tal processo é denominado de espiral do conhecimento, já que a criação do conhecimento organizacional é um processo em espiral, que começa no nível individual e vai ampliando comunidades de interação que cruzam fronteiras entre seções, departamentos, divisões e organizações (NONAKA; TAKEUCHI, 1997), conforme demonstra a Figura 1.

Figura 1 - Espiral do conhecimento

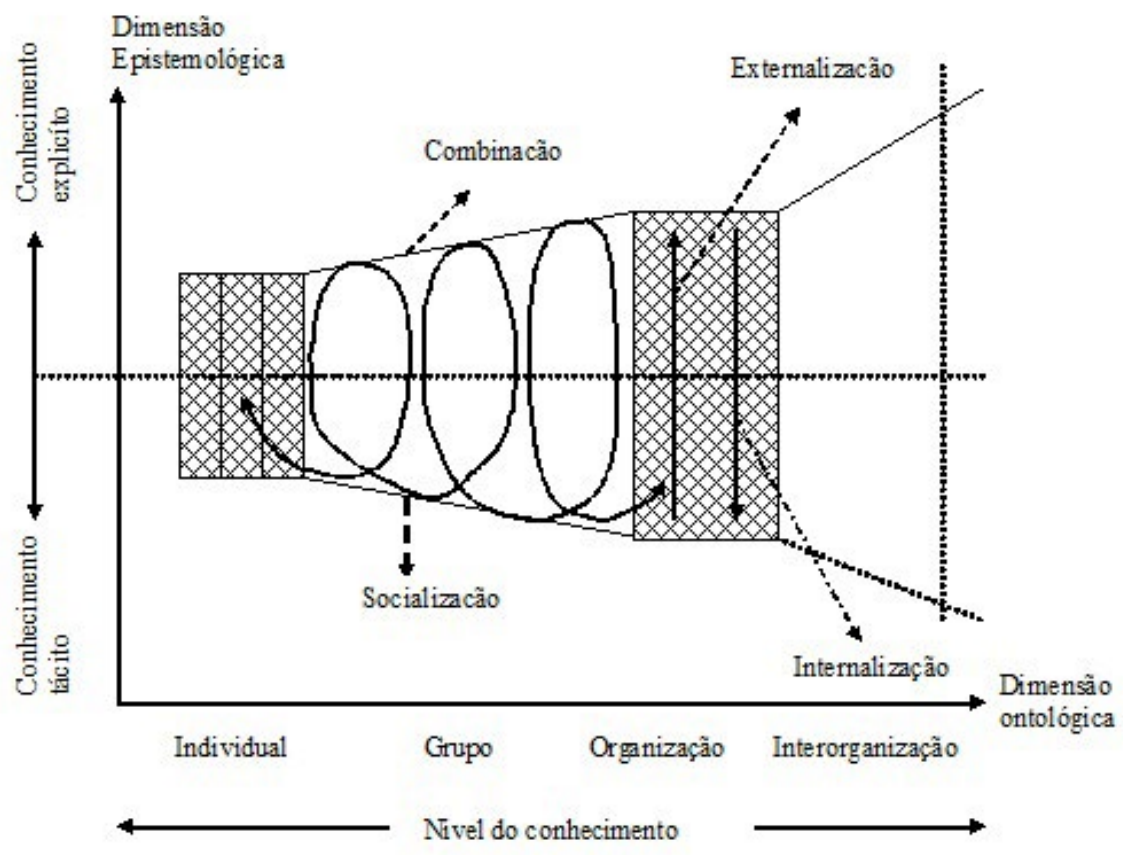

Fonte: Nonaka e Takeuchi (1997)

Rev. AdM. UfSM, SANTA MARIA, v. 8, NúMero 4, P. 650-665, OUT. - DEZ. 2015 


\subsection{Comunidades de prática}

A comunidade de prática pode ser definida como um grupo de pessoas auto-organizado e autogovernado que compartilha uma paixão a respeito de um domínio comum que mantém os participantes juntos (WENGER; SNYDER, 2001). Trata-se de grupos de indivíduos mantidos juntos de maneira informal, por meio de uma unidade compartilhada e unidade de propósitos, unidos em torno de um objetivo comum (PASCOTTO et al., 2013). As comunidades de prática são uma alternativa para a gestão do conhecimento organizacional (RIVERA, 2011).

Na perspectiva global, o conhecimento é considerado uma entidade dinâmica que é processada e analisada por meio de redes inteligentes que servem à sociedade do conhecimento. Nesse contexto, surge a comunidade de práticas, uma nova forma organizacional que complementa as estruturas existentes, possui ênfase no aprendizado e compartilhamento de conhecimentos e é baseada nas relações de confiança entre os membros. Por essa razão, os membros devem se adaptar ao comportamento da comunidade para buscar evolução de forma comum.

Dessa forma, a confiança representa um recurso eficiente para a coordenação no que diz respeito à flexibilidade do comportamento entre os membros (MARIES; SCARLAT, 2011). Para Binotto, Siqueira e Nakayama (2009), a confiança é construída no decorrer do tempo, à medida que as pessoas expõem suas ideias e participam da comunidade de prática.

O conceito de comunidades de prática tornou-se popular na gestão do conhecimento por melhorar as inter-relações sociais, por proporcionar aos membros do grupo compartilhar as melhores práticas e também por facilitar a transferência do conhecimento tácito (KRISHNAVENI; SUJATHA, 2012). Nas comunidades de prática, o conhecimento individual e as experiências são compartilhados, novos conhecimentos são desenvolvidos e problemas são resolvidos por meio das interações entre os membros. Visando ampliar o compartilhamento do conhecimento, as comunidades de prática estão se tornando virtuais (MARIES; SCARLAT, 2011).

Porém, a utilização de comunidades de práticas é um desafio social. Segundo Cady-Edmonds e Sosuisiti (2012), uma das dificuldades existentes consiste no fato de que essas comunidades não pertencem à organização e, por essa razão, não se subordinam à gerência da empresa, fato que demanda confiança e necessidade de os gestores apenas apoiarem as ações das comunidades.

Karlsen (2011), em seu estudo, analisou a microssociologia existente em comunidades de prática, de modo a favorecer o empreendedorismo e também a geração de inovações, assim como a ideia de que as comunidades de prática podem ser uma forma de inclusão, pois os processos que as geram favorecem o engajamento dos participantes na busca por soluções para um problema ou assunto específico. Segundo Lawthon (2011), as comunidades de prática despertam o sentimento de pertencimento em seus participantes, os quais precisam ser legitimados para poderem participar, pois todos compartilham valores, crenças e conhecimentos.

Para isso, é necessário entender a psicologia da comunidade e os caminhos que as empresas podem utilizar para trabalhar com essas comunidades de prática. A atual estrutura de tecnologia existente na internet faz com que essas comunidades sejam formadas fora das fronteiras organizacionais (KENDALL et al., 2010).

As comunidades de prática não são parte constituinte das organizações formais, existindo apenas pelo espírito de participação de seus membros e pelas conexões existentes entre todos eles. O engajamento mútuo é, assim, a base complementar das competências, ao conectar eficientemente o conhecimento dos membros (PRIMARD-GOGLIO, 2010). A importância das comunidades de prática reside, dessa forma, no fato de gerarem soluções mais rápidas e mais baratas para as demandas da organização (RIBEIRO; KIMBLE; CAIRNS, 2011). 


\section{MATERIAIS E MÉTODOS}

A escolha do setor de TI para este estudo ocorreu em função de o mercado mundial de software e serviços na área movimentar US\$1,4 trilhão, com previsão de mais de US\$ 3 trilhões para 2020 (BRASSCOM, 2012), além de empregar um grande contingente de mão de obra altamente especializada, que demanda metodologias de gestão do conhecimento. Devido ao fato de o setor de TI ser uma área intensiva em conhecimento, a capacitação e troca de informações são fatores estratégicos para as empresas e os profissionais dessa área, o que demanda o desenvolvimento de estudos que delineiem modelos de gestão do conhecimento voltados a atender às necessidades específicas de formação de mão de obra, fato que motivou o desenvolvimento deste estudo.

Para interrogar os respondentes, foi desenvolvido um instrumento de pesquisa composto de 18 variáveis e baseado na escala Likert, com 10 pontos, para melhorar a variabilidade dos dados, conforme demonstra o Quadro 1. O desenvolvimento do instrumento foi baseado na revisão da literatura e na avaliação de cinco especialistas, que analisaram a sua versão prévia e fizeram sugestões.

A coleta de dados ocorreu por meio de um levantamento transversal (BABBIE, 2011), ou seja, por meio de uma única coleta de dados com metodologia de amostragem por bola de neve (MALHOTRA, 2006). Para a seleção dos participantes, recorreu-se às redes sociais profissionais da internet (Linkedin, sites associações de profissionais da área de TI e gestão do conhecimento e grupos de discussão na internet). Os respondentes foram convidados a sugerir profissionais de seus relacionamentos que fossem analistas de sistemas, programadores e gestores de $\mathrm{Tl}$, com experiência mínima de um ano de atividades na área e participação em projetos de desenvolvimento de software, para participarem da pesquisa.

A amostragem do tipo bola de neve foi escolhida devido à dificuldade de formar uma amostragem representativa a partir dos órgãos oficiais, tais como a Relação Anual de Informações Sociais (RAIS) do Ministério de Trabalho e Emprego (MTE) e também o Sindicato dos TrabaIhadores em Informática e Tecnologia da Informação (SINDPD), em virtude de dois fatores: a) a grande maioria dos profissionais não possui relação formal com as empresas em que trabalham, justamente por se tratarem de projetos que possuem duração definida; e b) os salários pagos a esses profissionais superam em cerca de $70 \%$ o valor pago a um profissional registrado, o que faria com que os profissionais selecionados por amostragem probabilística em tais bases de dados não fossem representativos, distorcendo dessa forma as conclusões. Porém, devido à importância desse setor para o mercado brasileiro, que carece de formalização e também de estudos mais aprofundados, optou-se pelo desenvolvimento de um estudo quantitativo do tipo exploratório com uma amostra que fosse o mais representativa possível do perfil dos profissionais estudados, o que abre caminho para pesquisas mais aprofundadas e também de modelos mais precisos.

O período de coleta de dados foi de três meses (julho a setembro de 2008), em que foram enviados 500 questionários para profissionais de $\mathrm{TI}$, com o objetivo de obter pelo menos 185 questionários respondidos e válidos, pois, como não foi possível garantir a representatividade dos respondentes pelo critério de amostragem, utilizou-se o critério sugerido por Hair et al. (2005, p. 98) de que "o mínimo é ter pelo menos cinco vezes mais observações do que o número de variáveis a serem analisadas", índice que, na presente pesquisa, seria de 37 vezes 5, ou seja, 185. Entretanto, dos questionários respondidos, apenas 164 se enquadraram nos pré-requisitos para serem sujeitos da pesquisa, que são: profissionais de TI que atuam como programadores, analistas, administradores de bancos de dados, gerentes de projeto ou documentadores, e que prestam serviços em consultoria no desenvolvimento de softwares aplicativos na cidade de São Paulo. 
A amostra final da pesquisa pode ser considerada representativa para empresas de TI que desenvolvem sistemas aplicativos, devido ao fato de os questionários terem sido explicitamente direcionados a essa população, apesar da localização dos profissionais ter ocorrido com base em julgamentos em virtude da falta de uma estrutura amostral (lista de todos os possíveis respondentes). Em função dessa limitação, a pesquisa deve ser considerada exploratória, servindo como base para o conhecimento da população pesquisada e o desenvolvimento de futuras pesquisas para a generalização dos dados.

Os dados obtidos foram tabulados e analisados por meio da modelo de medidas PLS -PM, que é um conjunto de análises estatísticas destinadas a efetuar uma modelagem de equações estruturais (MEE) (HAIR, 2005), pois os dados não se mostraram aderentes a uma distribuição normal e o modelo geral causal tinha uma natureza mais exploratória. Ou seja, quando não se tem uma base teórica reconhecidamente consistente e consagrada, o PLS-PM é uma boa opção para a MEE (CHIN, 1995).

Quadro 1 - Instrumento de pesquisa

\begin{tabular}{|c|c|c|}
\hline & Variáveis & Questões \\
\hline \multirow{5}{*}{ 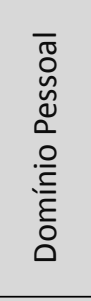 } & DP01 & Os profissionais são reconhecidos pela organização por trabalhos bem feitos. \\
\hline & DP02 & Os gestores sempre me incentivam e valorizam que eu faça novos cursos. \\
\hline & DP03 & $\begin{array}{l}\text { Sou incentivado o tempo inteiro pela organização para buscar novos conhecimen- } \\
\text { tos. }\end{array}$ \\
\hline & DP04 & Os profissionais são recompensados pela organização por trabalhos bem feitos. \\
\hline & DP05 & $\begin{array}{l}\text { A empresa permite e incentiva que os funcionários participem de fóruns de dis- } \\
\text { cussão. }\end{array}$ \\
\hline \multirow{4}{*}{ 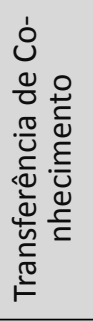 } & TC01 & $\begin{array}{l}\text { Todas as vezes em que tenho um problema técnico, procuro conversar com outros } \\
\text { membros da equipe. }\end{array}$ \\
\hline & TCO2 & $\begin{array}{l}\text { Os funcionários confiam uns nos outros e se sentem à vontade para compartilhar } \\
\text { conhecimentos e experiências. }\end{array}$ \\
\hline & TC03 & $\begin{array}{l}\text { Os gestores da organização valorizam atitudes de amizade entre os profissionais, o } \\
\text { que faz com que os profissionais confiem uns nos outros. }\end{array}$ \\
\hline & TCO4 & $\begin{array}{l}\text { Para compor equipes, os gestores sempre avaliam as competências técnicas e os } \\
\text { interesses dos profissionais. }\end{array}$ \\
\hline \multirow{5}{*}{ 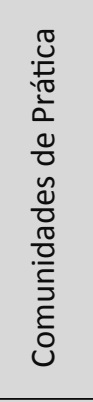 } & CP01 & $\begin{array}{l}\text { A organização permite e fornece ferramentas de comunicação aos funcionários } \\
\text { porque isso melhora o desempenho destes. }\end{array}$ \\
\hline & СP02 & $\begin{array}{l}\text { Os funcionários têm a percepção de que a organização possui um ambiente des- } \\
\text { contraído que valoriza a integração e a interação entre os funcionários. }\end{array}$ \\
\hline & СР03 & $\begin{array}{l}\text { A empresa estimula e valoriza manter grupos de amigos para compartilhar conhe- } \\
\text { cimentos e interesses a respeito de tecnologias ou outros assuntos. }\end{array}$ \\
\hline & СР04 & $\begin{array}{l}\text { Os gestores valorizam a opinião dos profissionais durante o processo de tomada } \\
\text { de decisão. }\end{array}$ \\
\hline & СP05 & $\begin{array}{l}\text { Minha percepção é de que os gestores acham o meu trabalho importante para a } \\
\text { organização. }\end{array}$ \\
\hline \multirow{4}{*}{ 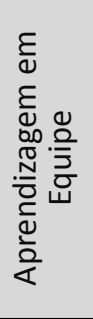 } & AE01 & $\begin{array}{l}\text { Os profissionais aceitam tranquilamente a ideia de outro profissional assumir a } \\
\text { liderança de determinado projeto. }\end{array}$ \\
\hline & AE02 & $\begin{array}{l}\text { Os empregados têm a percepção de possuir autonomia para desempenhar suas } \\
\text { tarefas e tomar decisões importantes. }\end{array}$ \\
\hline & AE03 & $\begin{array}{l}\text { A empresa vê com bons olhos o fato de eu consultar ou ser consultado pela minha } \\
\text { rede de amigos quando surgem dúvidas técnicas. }\end{array}$ \\
\hline & AE04 & $\begin{array}{l}\text { Tenho a percepção de que existe, na organização, uma forte relação de confiança } \\
\text { entre os funcionários que torna o trabalho em equipe possível. }\end{array}$ \\
\hline
\end{tabular}




\section{ANÁLISE DOS DADOS}

Devido ao fato de que o domínio pessoal necessita de um comprometimento pessoal para buscar o aprendizado (SENGE, 2001) e de que os profissionais de TI precisam trocar conhecimentos tácitos entre os membros de sua equipe ou de outras consultorias, o que caracteriza uma espiral do conhecimento segundo o modelo de Nonaka e Takeuchi (1997), a hipótese "H1" foi a seguinte:

H1 - “O domínio pessoal melhora a transferência de conhecimento entre os profissionais"

Entende-se que o domínio pessoal, proposto por Senge (2001), favorece o surgimento de comunidades de prática em empresas de $\mathrm{TI}$, devido à grande interação existente entre os membros das equipes de desenvolvimento ou suporte. Assim, a utilização de comunidades de prática auxilia as pessoas a colaborarem e também a adquirirem confiança nos membros da comunidade para adquirirem conhecimento, por meio de um aprendizado horizontal em rede (CADY-EDMONDS; SOSUISITI, 2012). Por esse motivo, a hipótese "H2" foi de que:

H2 - "O domínio pessoal melhora a participação e o número de comunidades de prática"

Por acreditar que o domínio pessoal facilita o trabalho de profissionais em equipe e, conforme apontado por Senge (2001), que a aprendizagem em equipe é vital, pois as equipes, e não os indivíduos, são a unidade de aprendizagem fundamental nas organizações modernas, a hipótese "H3" foi de que:

H3 - "O domínio pessoal melhora a aprendizagem em equipe"

A Figura 2, exposta a seguir, apresenta graficamente as hipóteses geradas.

Figura 2 - Hipóteses do modelo teórico de gestão do conhecimento

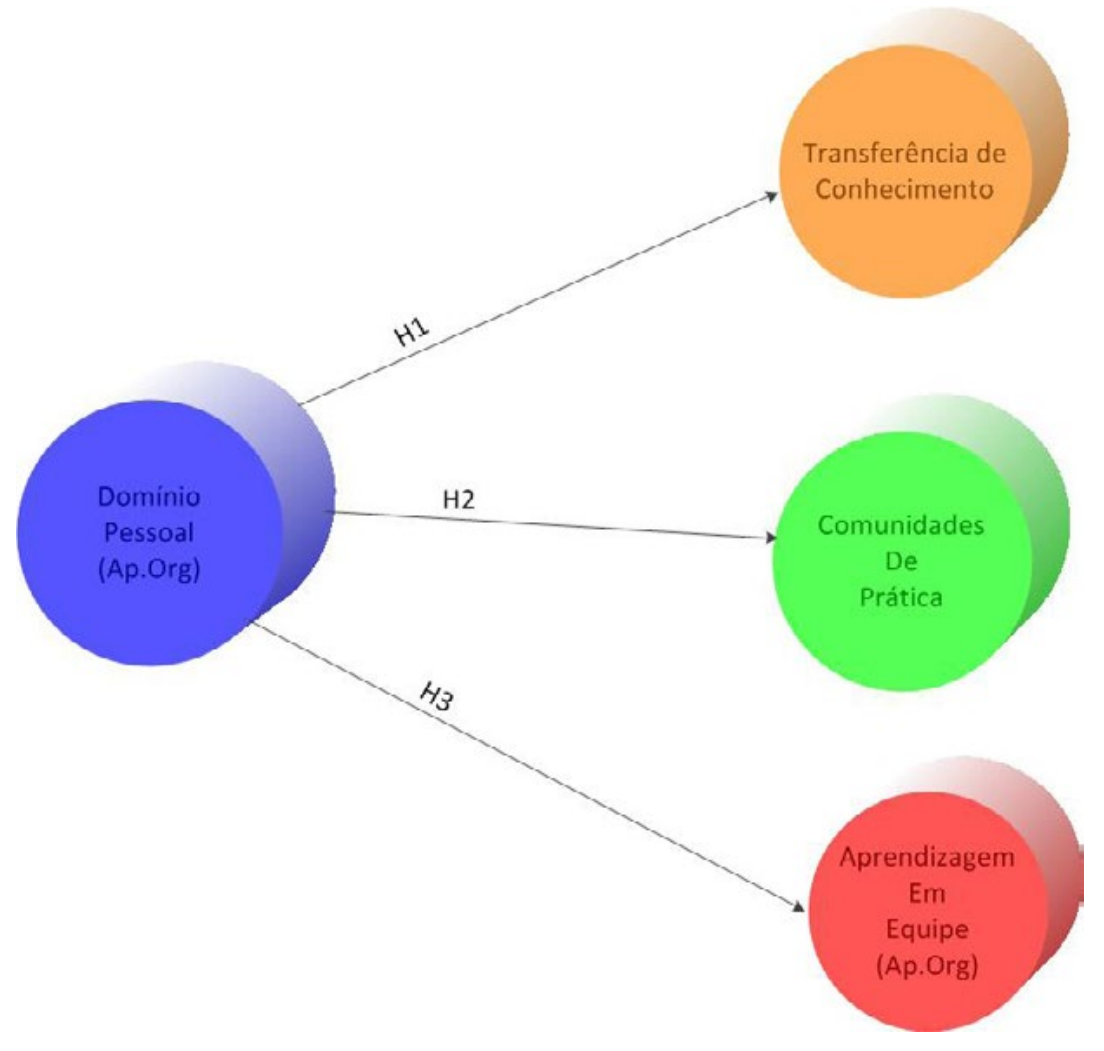

Rev. Adm. UFSM, SANTA MARIA, v. 8, NúMERo 4, P. 650-665, OUT. - DEZ. 2015 
Utilizando o modelo teórico apresentado na Figura 2, os dados foram processados na ferramenta SmartPIs 2.0, para efetuar a geração do modelo estatístico PLS-PM de gestão do conhecimento. Após a execução do software SmartPls, o modelo foi calculado. Primeiramente, procedeu-se à análise do chamado outer model, que é a relação entre as variáveis formativas que compõem o fator, observando-se a sugestão de que os valores de Average Variance Extracted (AVE) sejam de pelo menos 0,5 para cada variável (HAIR, 2010).

Após essa análise, as seguintes variáveis que apresentaram valores de AVE abaixo de 0,5 foram eliminadas do modelo, conforme a recomendação de Hair (2010):

AE02 $\rightarrow$ 0,098829, AE04 $\rightarrow-0,071049$ do fator aprendizagem em equipe;

CP05 $\rightarrow 0,220300$ do fator comunidades de prática;

DP02 $\rightarrow$ 0,184954, DP04 $\rightarrow$ 0,030181, DP05 $\rightarrow$ 0,285894 do fator domínio pessoal; e

TC01 $\rightarrow 0,105341$, TC03 $\rightarrow 0,013782$ do fator transferência do conhecimento.

Posteriormente, foram analisadas as correlações entre os fatores, por meio do R quadrado que apresentou baixo valor (0,094 e não 0,5, que é o valor mínimo recomendado) para a relação entre os fatores domínio pessoal e aprendizagem em equipe. Dessa forma, refutou-se a hipótese 3 , por não haver evidências de que o domínio pessoal melhora a aprendizagem em equipe.

O modelo de gestão do conhecimento final é apresentado na Figura 3, exposta a seguir.

Figura 3 - Modelo estatístico de gestão do conhecimento

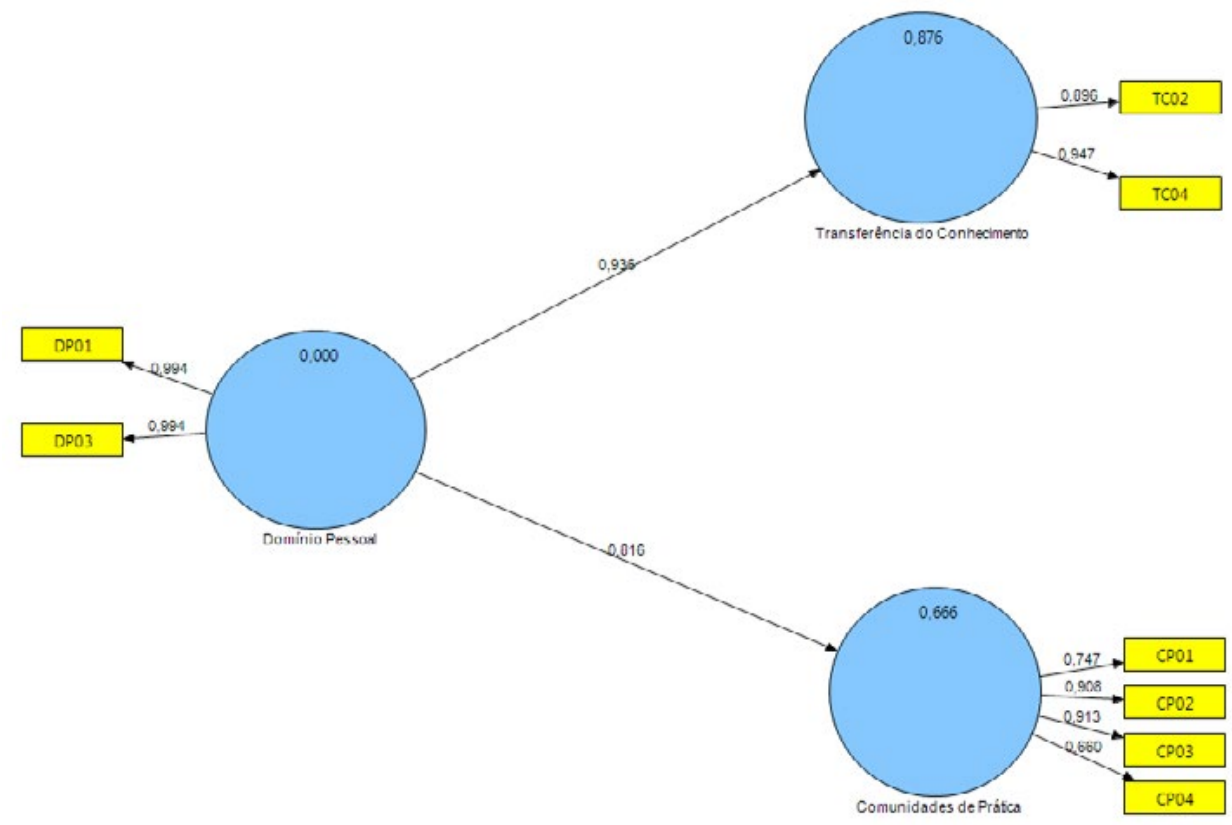

A Tabela 1 demonstra os valores do modelo de gestão do conhecimento, em que é possível verificar bons valores de AVE para os fatores, ou seja, valores acima de 0,5 (HENSELER; RINGLE; SINKOVICS, 2009), confiabilidade composta excelente para os três fatores e alpha de Cronbach considerado bom, isto é, acima de 0,6, que é o mínimo aceitável (HAIR et al., 2010). 
Tabela 1 - Valores do teste PLM-PM

\begin{tabular}{ccccc}
\hline & AVE & $\begin{array}{c}\text { Composite Relia- } \\
\text { bility }\end{array}$ & R Square & Cronbachs Alpha \\
\hline $\begin{array}{c}\text { Comunidades de } \\
\text { prática }\end{array}$ & 0,663045 & 0,88548 & 0,66596 & 0,822269 \\
Domínio pessoal & 0,987767 & 0,993846 & & 0,987615 \\
$\begin{array}{c}\text { Transferência do co- } \\
\text { nhecimento }\end{array}$ & 0,84908 & 0,918324 & 0,875807 & 0,82651 \\
\hline
\end{tabular}

A Tabela 2, a seguir, mostra as correlações existentes entre os fatores, indicando que o modelo escolhido apresenta bons índices de correlação, sendo o caminho escolhido o destacado (CHANG, 2011), e as raízes quadradas das AVEs apresentam (destacadas em cinza na Tabela 6) valores acima daqueles das correlações. Essa comparação mostra que o modelo geral tem uma boa validade discriminante (FORNELL; LARCKER, 1981).

Tabela 2 - Correlação entre os fatores e as raízes quadradas das AVEs

\begin{tabular}{cccc}
\hline & Comunidades de prática & Domínio pessoal & Transferência do conhecimento \\
\hline Comunidades de prática & 0,8142757518 & & \\
Domínio pessoal & 0,81606400 & 0,99386468 & \\
$\begin{array}{c}\text { Transferência do } \\
\text { conhecimento }\end{array}$ & 0,77696800 & 0,93584600 & 0,92145537 \\
\hline
\end{tabular}

A Tabela 3 demonstra os valores de cada variável que compõe os fatores, de forma que se torna possível avaliar a validade dos coeficientes de regressão. Segundo Hayduk (1987, p. 174), "o valor 1,96 (nível de significância de 5\%), é muito adequado para se garantir que cada caminho (ou carga) foi considerado como tendo validade preditiva".

Tabela 3 - Teste T por fator

\begin{tabular}{|c|c|c|c|}
\hline & Comunidades de prática & Domínio pessoal & $\begin{array}{c}\text { Transferência } \\
\text { do conhecimento }\end{array}$ \\
\hline СР01 & 1,780439 & & \\
\hline СР02 & 2,306069 & & \\
\hline СР03 & 2,286957 & & \\
\hline СР04 & 2,196892 & & \\
\hline DP01 & & 11,919818 & \\
\hline DP03 & & 13,388271 & \\
\hline TCO2 & & & 5,047049 \\
\hline TCO4 & & & 13,063859 \\
\hline
\end{tabular}

De acordo com a Tabela 3 e segundo Hayduk (1987), todas as variáveis são preditivas. $O$ passo seguinte foi medir o valor do Goodness of Fit (GoF). Para modelos em que todas as Variáveis Latentes (VL) são reflexivas, Tenenhuaus et al. (2005) propuseram um índice de adequação do modelo GoF, que basicamente é a média geométrica entre o R2 médio (adequação do modelo estrutural) e a AVE média (adequação do modelo de mensuração).

Para o modelo ora proposto, o valor encontrando foi 0,8478 , que é considerado um bom valor. Conforme Wetzels et al. (2009), o valor de 0,36 é adequado para as áreas de ciências sociais e do comportamento. 
O Quadro 2, exposto a seguir, demonstra as hipóteses que foram ou não confirmadas durante a análise PLS-PM. Pode-se notar que a hipótese 3 foi refutada por apresentar baixo valor de R2, indicando que a relação entre o fator domínio pessoal e aprendizagem em equipe é fraca ou inexistente. Provavelmente, esse índice baixo se deve à correlação entre dois fatores que estão ligados à aprendizagem organizacional.

Quadro 2 - Hipóteses do modelo

\begin{tabular}{|l|l|c|}
\hline H1 & O domínio pessoal melhora a transferência de conhecimento entre os profissionais & Confirmada \\
\hline H2 & O domínio pessoal melhora a participação e o número de comunidades de prática & Confirmada \\
\hline H3 & O domínio pessoal melhora a aprendizagem em equipe & Não confirmada \\
\hline
\end{tabular}

\section{ANÁLISE DOS RESULTADOS E DISCUSSÕES}

O objetivo deste estudo foi atingido ao se desenvolver um modelo de gestão do conhecimento para profissionais de TI. Este estudo parte do pressuposto de que o domínio pessoal é o pilar da gestão do conhecimento para profissionais da área, pois pessoas com um alto nível de domínio pessoal conseguem realizar mais rapidamente aquilo que é mais importante para elas.

Pode-se concluir, dessa forma, que a capacidade e o comprometimento de uma organização em aprender não devem ser maiores que a de seus integrantes. Porém, surpreendentemente poucas organizações estimulam o crescimento de seus funcionários, fato que se mostra verdadeiro para profissionais de TI, pois, devido ao grande número de softwares e metodologias que necessitam aprender durante o desenvolvimento de projetos, o ritmo de estudo e de aprendizado imposto é muito alto, fato que às vezes impede a busca de treinamentos formais.

Além disso, algumas empresas alocam os profissionais em projetos durante um período, cerca de seis meses, intercalando com períodos de treinamentos formais. Em alguns casos, as consultorias mantêm os profissionais em projetos, mas incentivam a troca de conhecimentos entre os profissionais.

Devido a esse fator, a primeira hipótese foi de que o domínio pessoal favorece a transferência de conhecimento, uma vez que trocar conhecimentos envolve confiança e a existência de uma base de know-how para trocar com os seus pares. Como o principal ativo desses profissionais é know-how, ou seja, conhecimento tácito, essa hipótese foi confirmada. Há, entretanto, dificuldade para criar documentações que captem as necessidades de conhecimentos dos profissionais, já que a maioria dos projetos envolve situações únicas.

Tseng et al. (2012) afirmam que a transferência do conhecimento é um aspecto derivado da gestão do conhecimento que amplia as suas ações, pois possibilita que o conhecimento seja distribuído para outras empresas, atingindo áreas geográficas maiores. A confirmação desta hipótese está de acordo com o modelo de Nonaka e Takeuchi (1997), que parte do princípio de que o conhecimento é criado em duas dimensões, a epistemológica e a ontológica.

Por essa razão, Lichtenthaler (2010) sugere que algumas empresas enfrentam dificuldades no que se refere à transferência de conhecimento, aspecto que se deve em boa parte ao uso de estruturas formais e hierárquicas, o que dificulta a transferência do conhecimento. Esse é um dos fatores que levaram à elaboração da segunda hipótese - o domínio pessoal favorece o surgimento das comunidades de prática, uma ferramenta de transferência de conhecimento informal, que é bastante utilizada por profissionais de TI, porém de forma inconsciente.

Para Wenger e Snyder (2001), uma comunidade de prática é um grupo de pessoas auto-organizado e autogovernado que compartilha uma paixão a respeito de um domínio comum 
que mantém os participantes juntos. Trata-se de uma alternativa para a gestão do conhecimento organizacional (RIVERA, 2011).

Os profissionais de $\mathrm{TI}$ valem-se de comunidades de prática, por meio das redes pessoais de relacionamento, principalmente para tirar dúvidas ou trocar informações. Existem, também, ações por parte de fabricantes que geram fóruns de discussão e que trazem notoriedade para os profissionais que ajudam na solução de dúvidas. Um exemplo disso é a Microsoft, que atribui pontuações para os profissionais que solucionam dúvidas de outros profissionais a respeito de seus produtos. Outro exemplo é a Oracle, que incentiva os profissionais a produzirem documentações e artigos a respeito dos seus produtos, premiando-os com uma certificação chamada Oracle Ace, de grande notoriedade e bastante valorizada pelo mercado.

No entanto, as empresas devem entender que as comunidades de prática perpassam suas fronteiras organizacionais e, por essa razão, não se subordinam a sua gestão. Diante disso, é preciso incentivar e favorecer o acesso dos profissionais a tais comunidades, para colher os benefícios dessa relação.

Tais questões motivaram a elaboração da terceira hipótese: o domínio pessoal favorece a aprendizagem em equipe. Essa hipótese não foi confirmada, talvez por tratar de dois aspectos ligados à aprendizagem organizacional, o que certamente leva a crer que as duas práticas (domínio pessoal e aprendizagem em equipe) devem ser trabalhadas juntas, conforme sugere Senge (2001). Talvez por essa razão fosse prudente, em outros estudos, tratar o fator domínio pessoal como aprendizagem organizacional e não mais como apenas um aspecto de tal disciplina.

A utilização das iniciais dos aspectos domínio pessoal, transferência de conhecimento e comunidades de prática motivou os pesquisadores a nomearem o modelo proposto neste estudo de DTC, que representa os fatores que devem ser gerenciados para a gestação do conhecimento em empresas de TI.

\section{CONSIDERAÇÕES FINAIS}

O objetivo deste trabalho foi atingido ao se desenvolver e testar, ainda que em uma amostra obtida por julgamento, o modelo de gestão do conhecimento para consultoria de TI. A motivação para este estudo surgiu da necessidade de gestão do conhecimento que esse tipo de organização necessita, uma vez que estas desenvolvem diversos projetos que, na maioria das vezes, são únicos e nem sempre possuem toda a expertise necessária para desenvolvê-los.

Portanto, os profissionais da área participantes desses projetos são formados durante o seu próprio desenvolvimento, gerando, dessa forma, a necessidade de um comprometimento de aprendizado, que deveria ser apoiado pelas organizações. Por essa razão, o modelo desenvolvido neste estudo parte do pressuposto de que o primeiro aspecto a ser observado é o domínio pessoal, indo ao encontro do modelo proposto por Nonaka e Takeuchi (1997), que pressupõe que o conhecimento nasce no indivíduo e depois é disseminado pela organização e, posteriormente, para fora dela.

Neste estudo, percebeu-se forte correlação entre o domínio pessoal proposto por Senge (2001) e a transferência do conhecimento. Sendo assim, as organizações devem oferecer mecanismos e estruturas que possibilitem e favoreçam essa troca, tais como ambientes que facilitem as reuniões e as discussões, conforme já observado por Lichententhaler (2010).

Outro aspecto importante é a forte correlação percebida entre o domínio pessoal e o surgimento de comunidades de prática. Isso se deve principalmente às dificuldades que os profissionais possuem em obter conhecimentos na velocidade em que o projeto demanda, processo facilitado pelo surgimento de comunidades que favorecem a troca e a interação entre os 
profissionais da organização e de outras empresas, como apontado por Rivera (2011). O uso de comunidades de prática favorece o surgimento de inovações e a resolução de problemas, aspecto importante em projetos de TI, corroborando com o proposto por Wenger e Snyder (2001).

Já a hipótese 3 foi refutada, talvez devido ao fato de que relaciona dois aspectos ligados à aprendizagem organizacional que precisariam, por essa razão, serem gerenciados de forma integrada e não de forma separada como sugeriu este estudo. Nesse sentido, futuros estudos devem considerar o fator domínio pessoal como aprendizagem organizacional, o que corrobora as afirmações propostas por Senge (2001), de que as disciplinas devem ser utilizadas de forma integrada e não de forma separada.

A importância deste trabalho reside, assim, no fato de ter desenvolvido um modelo que busca representar a gestão do conhecimento no setor de projetos de $\mathrm{TI}$, por meio da análise de dados oriundos de uma população representativa desse setor. Em futuros estudos, os pesquisadores podem gerar um novo modelo teórico, partindo da hipótese de que a aprendizagem organizacional é o fator que deve servir como ponto de partida por influenciar os demais fatores.

Este estudo também pode ser aplicado a outros públicos para verificar a consistência do modelo, já que empresas de TI pressupõem um ambiente propício para o desenvolvimento das práticas de gestão do conhecimento. Seria importante, ainda, aplicar o estudo em outras regiões, já que todos os profissionais participantes pertenciam a empresas sediadas somente em São Paulo.

\section{REFERENCIAS}

AKHTAR, N.; KHAN, R. A. Exploring the Paradox of Organization Learning. Interdisciplinary Journal of Contemporary Research in Business, Janeiro, 2011. 257-270.

ASLAM, D. H.; JAVID, T.;TANVEER, A.; KHAN, M.; SHABBIR, F. Journey from Individual to Organization Learning. International Journal of Academic Research, Maio, 2011. 738-745.

BABBIE, E. Métodos de Pesquisa de Survey. Belo Horizonte: Editora UFMG, 2011.

BINGHAN, C. B.; DAVIS, J. P. Learning Sequences: Their Existence, Effect and Evolution. Academy of Management Journal, v.55, n.3, p.611-641, 2012.

2012, Vol. 55, No. 3, 611-641.

BINOTTO, E; SIQUEIRA, E. S; NAKAYAMA, M. K. Criação de Conhecimento no Agronegócio: estudo de casos. Revista de Administração da UFSM, Santa Maria, v. 2, n. 3, p. 367-384, 2009.

BRASSCOM. Quatro Desafios. Disponível em <http://www.brasscom.org.br/brasscom/box opinion/opinion>. acesso em 06/10/2012, 11:40:00.

CADY-EDMONDS, C.; SOSUISITI, M. R. Aplications of Situated Learning to Foster Communities of Practice, v. 48, n. 1, p. 45-64, 2012.

CHANG, C. H. The Influence of User's Trait on Public e-Service Usage:A Self-Service Technology Perspective. Asian Social Science, Tokio, v.7, n.7, p. 3-11, 2011.

CHIN, W. W. Partial Least Squares is to LISREL as principal components analysis is to common factor analysis. Technology Studies, v.2, p. 315319, 1995.

DAVENPORT, T.; PRUSAK, L. Conhecimento Empresarial: como as organizações gerenciam o seu capital intelectual. 8. ed. Rio de Janeiro: Campus, 1998.

FORNELL, C.; LARCKER, D. F. Evaluating structural equation models with unobservable variables and measurement error. Journal of Marketing Research, v.18, n.1, p.39-50, 1981. 
GIL, A. C. Métodos e Técnicas de Pesquisa Social. 5. ed. São Paulo: Atlas, 2006.

HAIR, J. et al. Multivariate Data Analysis. 7. ed. [S.I.]: Pearson Prentice Hall, 2010.

HAIR, J. F. E. A. Análise Multivariada de Dados. 5. ed. Porto Alegre: Bookman, 2005.

HAYDUK, L. Structural equation modeling with LISREL. Baltimore: The Johns Hopkins University Press, 1987.

HENSELER, J.; RINGLE, C. M.; SINKOVICS, R. R. The use of partial least squares path modeling in international marketing. Advances in International Marketing, v.20, p. 277-319, 2009.

IMRAN, M.; RISVI, S. H. M.; ALIZ, B. Impact of Organizational Learning Earning on Organizational Performance. International Journal of Academic Research, v.3, n.5, p. 424427, 2011.

KARLSEN, A. Cluster Creation by Reconfiguring Communities of Practice. European Planning Studies, v. 19, n. 5, p. 753-773, Mai. 2011.

KENDALL, $\mathrm{H}$. et al. Eletronic Communities of Practice: Guidelines From a Project. Journal of Continuing Education in the Health Professions, v. 30, n. 2, p. 139-143, 2010.

KILBRIDE, C. et al. Developing theory and practice: Creation of a Community of Practice through Action Research Produced Excellence in Stroke Care. Journal of Interprofessional Care, v. 25, p. 91-95, 2011.

KRISHNAVENI, R.; SUJATHA, R. Communities of Practice: An Influencing Factor for Effective Knowledge Transfer in Organizations. The IUP Journal of Knowledge Management, v. 10, n. 1, p. 26-40, 2012.

LAWTHON, R. Developing Learning Communities: using communities of practice within psychology. International Jounal of Inclusive Education, v. 15, n. 1, p. 153-164, Fev.
2011.

LEITE, N. R. P; ALBUQUERQUE, L. G. A. Gestão Estratégica de Pessoas, Estratégia de Retenção de Profissionais e Comprometimento OrganizacionalemumaEstruturaOrganizacional Remota. Revista de Administração da UFSM, Santa Maria, v. 6, n. 4, p. 803-822, Dez. 2013. DOI: $10.5902 / 198346597217$.

LICHTENTHALER, U. Outward Knowledge Transfer: The Impact of Project-based Organization on Performance. Industrial and Corporate Change, v.19, n.6, p. 1705-1739, Jul. 2010.

MALHOTRA, N. Pesquisa de Marketing: uma Orientação Aplicada. 4a $\underline{a}$ ed. Porto Alegre: Bookman, 2006.

MARIES, J.; SCARLAT, E. Applying a Computational Trust and Reputation Model of Communities of Practice. Review of Economic Studies \& Research, v. 4, n. 2, p. 59-77, Dezembro 2011.

MOHANTY, K.; KAR, S. Achieving Innovation and Sucess: Organizational Learning. SCMS Journal of Indian Management, v.9, n.1, p.3642, Jan. 2012.

NONAKA, I.; TAKEUCHI, H. Criação do Conhecimento na Empresa. 13. ed. Rio de Janeiro: Elsevier, 1997.

O'LEARY, M. B.; MORTENSEN, M. Multiple Team Membership: A Theoretical Model of its Effects on Productivity and Learning for Individuals and Teams. Academy of Management Review, v.36, n.3, p.461-478, Jul. 2011.

OSBORN, E.; DAWSON, S. Learning accross Communities of Practice: An Examination of Multidisciplinary Work. British Journal of Management, v. 21, n.4, p. 843-858, 2010.

PASCOTTO, S. M. P; FARINA, M. C; TELMO, T. H. P; DUGO, J. C. Análise de Rede Social para mensuração das estruturas formais e informais. Revista de Administração da UFSM, 
Santa Maria, v. 6, Edição Especial, p. 179-194, Mai. 2013. DOI: 10.5902/198346598808.

PHIPPS, S. T. A.; PRIETO, L. C.; VERMA, S. Holding the Helm: Exploring the Influence of Transformational Leadership on Group Creativity, and the Moderating Role of Organizational Learning Culture. Journal of Organizational Culture, v.16, n.2, p.145-157, 2012.

PRIMARD-GOGLIO, K. Le role d'un système communautaire, object-frontière entre deus communautés de pratique d'ingénieurs développement. Revue Gestion 2000, v. 27, n. 4, p. 91-111, Jul-Out. 2010.

RIBEIRO, R.; KIMBLE, C.; CAIRNS, P. Some first steps in the search of hidden Communities of Practice within eletronic Networks. Journal of Organisational Transformation and Social Change, v. 8, n. 2, p. p.183-197, 2011.

RIVERA, J. C. A. Communities of Practice: Improving Knowledge Management in Business. Business Education \& Accreditation, v. 3, n. 3, p. 102-111, 2011.

RODRIGUES, P; PEDRON, C.D; OLIVEIRA, $M$. Knowledge Management on the implementation of a CRM Project: A Case Study. Revista de Administração da UFSM, Santa Maria, v. 6, número 3, p. 595-613, Set. 2013. DOI: $10.5902 / 198346598486$.

SENGE, P. A Quinta Disciplina - Arte e Prática da Organização de Aprendizagem. 9. ed. São Paulo: Best Seller, 2001.

SRIHAWONG, J.; SRISA-ARD, B. Development of the Learning Organization Model: A Case of Sisaket Rajabhat University. European Journal of Social Sciences, v.27, n.12, p. 233-241, 2012.

STACKE, A. R. N. P.; HOFFMAN, V. E.; COSTA, H. A. Knowledge Trasnfer Among Clustered Firms: A Study of Brazil. Anatolia - An International Journal of Tourism, v.23, n.1, p. 90-106, Abr. 2012.
TAHIR, A.; NAEEM, H.; SARFRAZ, N.; JAVED, A.; $A L I, R$. Organizational Learning and Employee Performance. Institute of Interdisciplinary Business Research, v.3, n.2, p.1506-1514, Jun. 2011.

TSENG, K. H.; CHANG, C.C.; LOU, S.J.; TAN, Y.; CHIU, C.J . Concept-mapping Perception Navigates Student Knowledge Transfer. Educational Technology \& Society, v.15, n.1, p.102-15, 2012.

USSAHAWANITCHAKIT, P. Organizational Learning and Organization Innovation: Evidence from Eletronics Business in Thailand. International Journal of Business Research, v.11, n.2, p.137-145, nov. 2011. 137-145.

WANG, P.; RAMILLER, N. C. Community Learning in Information Technology Innovation. MIS Quartely, v.33, n.4, p. 709-734, Dez. 2009.

WENGER, E. C.; SNYDER, W.M. Comunidades de prática: a fronteira organizacional. In: HARVARD BUSINESS REVIEW (Org.). Aprendizagem Organizacional. Tradução de Cásia Maria Nasser. Rio de Janeiro: Campus, 2001.

WETZELS, M. E. A. Using PLS path modeling for assessing hierarchical construct models: guidelines and empirical illustration, v. 33, n. 1, p. 177-195, Mar. 2009. 